\title{
Joint Beamforming And Power Splitting Design For C-RAN With Multicast Fronthaul
}

\author{
Wanming Hao, Gangcan Sun, Ming Zeng, Zhengyu Zhu, Bin Jiang, and Shouyi Yang
}

\begin{abstract}
In this paper, we investigate the joint beamforming and power splitting design problem in a base station (BS) clusterbased cloud radio access network (C-RAN) with multicast fronthaul, where users are jointly served by BSs within each cluster. Meanwhile, each user can simultaneously obtain information and energy from received signals. On this basis, under predefined minimum harvested energy of each user and maximum transmit power of each BS and central processor, we formulate a sum rate maximization problem by jointly optimizing multicast fronthaul beamforming, cooperative access beamforming and power split ratios. Due to the difficulty in solving the formulated problem, we first transform it into a convex one by successive convex approximate and semidefinite program (SDP) relaxation techniques, and then propose an effective iterative algorithm. Moreover, we design a randomization method that can always obtain the rankone solution. Finally, numerical results are conducted to validate the effectiveness of our proposed algorithm.
\end{abstract}

Index Terms-SWIPT, C-RAN, Multicast.

\section{INTRODUCTION}

To satisfy the increasing demands of the data rate in future mobile networks, ultra-dense base station (BS) deployment is deemed as one of effective schemes [1]. However, this leads to more serious interference among BSs. To handle it, cloud radio access network (C-RAN) structure is developed, where each BS is connected to the central processor $(\mathrm{CP})$ [2]. The CP jointly manages the interference by global resource allocation, effectively improving the spectral efficiency (SE) and relieving the BSs' burden (via moving the baseband processing to the baseband unit (BBU) pool) [3]. However, the C-RAN structure brings another challenge, i.e, the selection of the fronthaul links carrier. Traditionally, wired fronthaul link is adopted due to its high stability and capacity. Nonetheless, its high deployment cost makes it unsuitable for the ultra-dense BS, and thus, the wireless carrier becomes a suitable candidate [4]. In addition, coordinated multiple-point (CoMP) transmission technology is also an effective approach to remove the adjacent-BS interference, which has been widely applied in C-RAN, e.g., [4][6]. Since the cooperative BSs need to jointly serve users,

W. Hao is with the School of Information Engineering, and the Henan Institute of Advanced Technology, Zhengzhou University, Zhengzhou 450001, China, and also with the National Center for International Joint Research of Electronic Materials and Systems, Zhengzhou 450001, China. (Email: iewmhao@zzu.edu.cn).

G. Sun, Z. Zhu, and S. Yang are with the School of Information Engineering, Zhengzhou University, Zhengzhou 450001, China. (E-mail: \{iegcsun, iezyzhu, iesyyang\}@zzu.edu.cn)

M. Zeng is with the Faculty of Science and Engineering, Laval University, Quebec, G1V0A6, Canada, and also with the Faculty of Engineering and Applied Science, Memorial University, St. Johns, NL A1B 3X9, Canada. (Email: mzeng@mun.ca).

B. Jiang is with the College of Information and Communication, National University of Defense Technology, Wuhan 430010, China. (E-mail: jiangbin17@nudt.edu.cn) the CP should transmit each user's signal to these BSs, i.e., point-to-multipoint transmission. To realize the above, multicast technique will be a suitable scheme, which has been adopted in [4] and [6]. Therefore, to further enhance the performance of system, CoMP transmission-based C-RAN with multicast fronthaul represents a promising solution.

On the other hand, the simultaneous wireless information and power transfer (SWIPT) technique is developed for improving energy efficiency, where both information and energy are extracted from the same received RF signals [7]. Although a lot of recent works investigate the SWIPT, e.g., [8]-[10], the study under C-RAN structure is limited. The authors in [11] consider the weighted sum rate maximization for a multiuser multiple-input multiple-output SWIPT system, where the beamformers in both the downlink and uplink, and the time allocation are jointly optimized. The authors in [12] consider a transmit power minimization problem in a full duplex C-RAN, and four approaches of jointly optimizing beamformers, uplink transmit power and receiver power ratios are proposed. The authors in [13] study the min max fronthaul load optimization problem for an energy harvesting powered CRAN with QoS and harvested energy constraints, and propose an effective beamforming algorithm to solve it. In [14], the authors investigate SWIPT problem in an uplink C-RAN, and the minimum mean-square-error is considered by optimizing precoders and detectors. [15] considers an energy-efficient uplink resource allocation problem by optimizing the subcarrier and power allocation, and then, a quantum-behaved particle swarm-based low-computational suboptimal algorithm is proposed. The max-min fair SWIPT is investigated in a green C-RAN with millimeter wave (mmWave) fronthaul [16]. The minimum data rate is maximized via a two-step iterative bemaforming algorithm. Although [12]-[16] involve the SWIPT in C-RAN, the cooperation among multiple BSs is not considered. Therefore, the multicast fronthaul and CoMP techniques are not investigated, including the multicast beamforming and cooperative beamforming design.

Unlike the previous works, in this paper, we consider a downlink BS cluster-based SWIPT C-RAN with multicast fronthaul, where multiple BSs jointly serve users within one cluster. In general, the fronthaul link distance between the $\mathrm{CP}$ and the BS cluster is relatively large, and thus, adopting mmWave is not appropriate due to its large path loss. Therefore, microwave (current cellular frequency, e.g., sub-6 GHz) is adopted owing to its small path loss. In contrast, the access link distance between the BS cluster and users is small, and thus, using mmWave is appropriate. Specifically, the CP transmits data to each BS cluster via microwave multicast fronthaul links, and users simultaneously receive information and energy from the 
BS via mmWave access links. Based on this, we formulate a sum rate maximization problem by jointly optimizing multicast fronthaul beamforming, cooperative access beamforming and power split ratios, under maximum transmit power constraint for each BS and the CP. For the formulated non-convex optimization problem, we first transform it into a semidefinite program (SDP) optimization problem. Then, via successive convex approximate (SCA) and SDP relaxation techniques, the SDP problem is relaxed into a convex one, and an iterative algorithm is proposed. Finally, we propose a rank-one solution based on the randomization method.

\section{System Model and Problem Formulation}

We consider a downlink C-RAN with one CP and $L$ BS clusters. The $\mathrm{CP}$ is equipped with $N$ antennas, while each BS is mounted with one antenna for transmitting mmWave signal and receiving microwave signal simultaneously. We assume that there are $M$ BSs and $K$ users in each cluster, and each user is jointly served by $M$ BSs. It is assumed that each user is equipped with power splitter hardware that can split the received signal into the information decoder (ID) and energy harvester $(\mathrm{EH})$. In addition, the beamforming and power splitting design takes place at the CP.

The received signal at $\mathrm{BS}(l, m)$ can be expressed as

$$
y_{l m}^{\mathrm{FH}}=\mathbf{h}_{l m} \mathbf{v}_{l} x_{l}+\sum_{j \neq l}^{L} \mathbf{h}_{l m} \mathbf{v}_{j} x_{j}+n_{l m}
$$

where $\mathrm{BS}(l, m)$ denotes the $m$ th BS of the $l$ th cluster, $\mathbf{h}_{l m} \in$ $\mathbb{C}^{1 \times N}$ represents the fronthaul link channel coefficient from the CP to BS $(l, m), \mathbf{v}_{l} \in \mathbb{C}^{N \times 1}$ is the multicast beamforming for the $l$ th cluster, and $x_{l}$ is the multicast signal with $\mathbb{E}\left\{\left|x_{l}\right|^{2}\right\}=1 . \mathbf{n}_{l m}$ is an independent and identically distributed (i.i.d.) additive white Gaussian noise (AWGN), where each entry follows $C \mathcal{N}\left(0, \delta^{2}\right)$.

To this end, the achievable fronthaul rate can be written as

$$
R_{l m}^{\mathrm{FH}}=B_{\mathrm{mc}} \log \left(1+\frac{\left|\mathbf{h}_{l m} \mathbf{v}_{l}\right|^{2}}{\sum_{j \neq l}^{L}\left|\mathbf{h}_{l m} \mathbf{v}_{j}\right|^{2}+B_{\mathrm{mc}} \delta^{2}}\right),
$$

where $B_{\mathrm{mc}}$ denotes the microwave bandwidth. The multicast rate of the $l$ th cluster is decided by the BS with the worst channel condition, and thus the fronthaul multicast rate provided by the CP for the $l$ th cluster is given [13]

$$
R_{l}^{\mathrm{FH}}=\min _{m \in \mathcal{M}}\left\{R_{l m}^{\mathrm{FH}}\right\}, l \in \mathcal{L},
$$

where $\mathcal{M}=\{1, \cdots, M\}$ and $\mathcal{L}=\{1, \cdots, L\}$ denote the BS and cluster sets, respectively.

The received signal of User $(l, k)$ can be expressed as

$$
y_{l k}^{\mathrm{AC}}=\mathbf{g}_{l l k} \mathbf{w}_{l k} x_{l k}+\sum_{i \neq k}^{K} \mathbf{g}_{l l k} \mathbf{w}_{l i} x_{l i}+\sum_{j \neq l}^{L} \sum_{i=1}^{K} \mathbf{g}_{j l k} \mathbf{w}_{j i} x_{j i}+n_{l k},
$$

where User $(l, k)$ denotes the $k$ th user of the $l$ th cluster, $\mathbf{g}_{j l k}=$ $\left[g_{j l k}^{1}, \cdots, g_{j l k}^{M}\right]$ represents the downlink channel coefficient from $M$ BSs of the $j$ th cluster to User $(l, k), g_{j l k}^{m}$ denotes the downlink channel coefficient from $\mathrm{BS}(j, m)$ to User $(l, k)$. In addition, $\mathbf{w}_{l k} \in \mathbb{C}^{M \times 1}$ and $x_{l k}$, respectively, denote the cooperative beamforming and signal for User $(l, k)$, and $\mathbf{n}_{l m}$ is an i.i.d. AWGN with $\operatorname{CN}\left(0, \delta^{2}\right)$. In (4), the first term stands for the desired signal, the second term is the intra-cluster interference, and the third term represents the inter-cluster interference.

The received signal at each user are divided into two parts, i.e., ID and EH. Let $\beta_{l k}$ denote the power splitting factor for User $(l, k)$, the received signal for the ID can be expressed as

$$
y_{l k}^{\mathrm{ID}}=\sqrt{\beta_{l k}} y_{l k}^{\mathrm{AC}}+u_{l, k}
$$

where $u_{l, k}$ denotes the caused noise due to the power splitting and follows $C \mathcal{N}\left(0, \delta_{u}^{2}\right)[8]$. Accordingly, the achievable rate of User $(l, k)$ can be written as

$$
R_{l k}^{\mathrm{AC}}=B_{\mathrm{mm}} \log \left(1+\gamma_{l k}\right)
$$

where $B_{\mathrm{mm}}$ is downlink mmWave bandwidth, and

$\gamma_{l k}=\frac{\left|\mathbf{g}_{l l k} \mathbf{w}_{l k}\right|^{2}}{\sum_{i \neq k}^{K}\left|\mathbf{g}_{l l k} \mathbf{w}_{l i}\right|^{2}+\sum_{j \neq l}^{L} \sum_{i=1}^{K}\left|\mathbf{g}_{j l k} \mathbf{w}_{j i}\right|^{2}+B_{\mathrm{mm}} \delta^{2}+\delta_{u}^{2} / \beta_{l k}}$.

In addition, the received signal for the EH can be expressed as

$$
y_{l k}^{\mathrm{EH}}=\sqrt{1-\beta_{l k}} y_{l k}^{\mathrm{AC}},
$$

and the harvested energy is

$$
E_{l k}=\eta\left(1-\beta_{l k}\right)\left(\sum_{j=1}^{L} \sum_{i=1}^{K}\left|\mathbf{g}_{j l k} \mathbf{w}_{j i}\right|^{2}+B_{\mathrm{mm}} \delta^{2}\right)
$$

where $\eta$ denotes the energy conversion efficiency at each user.

\section{A. Problem Formulation}

In this paper, our objective is to maximize the sum rate by jointly optimizing beamforming and power split ratios, which can be formulated as

$$
\begin{aligned}
\max _{\left\{\mathbf{v}_{l}, \mathbf{w}_{l, k}, \beta_{l k}\right\}} & \sum_{l=1}^{L} \sum_{k=1}^{K} R_{l k}^{\mathrm{AC}} \\
\text { s.t. } & E_{l k} \geq E_{l k}^{\min }, \forall l, k, \\
& \sum_{l=1}^{L}\left\|\mathbf{v}_{l}\right\|^{2} \leq P_{\mathrm{CP}}^{\mathrm{max}}, \\
& \sum_{k=1}^{K}\left|\mathbf{w}_{l, k}(m)\right|^{2} \leq P_{l, m}^{\max }, \forall l, m, \\
& \sum_{k=1}^{K} R_{l k}^{\mathrm{AC}} \leq R_{l}^{\mathrm{FH}}, \forall l,
\end{aligned}
$$

where (9b) denotes the minimum harvested energy for each user, 9c and 9d represent the maximum transmit power constraints for the CP and BS $(l, m)$, respectively, where $\mathbf{w}_{l, k}(m)$ is the $m$ th element of $\mathbf{w}_{l, k}$, and $9 \mathrm{e}$ is the fronthaul capacity constraint. Due to the non-convex objective function $9 \mathrm{a}$, constraints $9 \mathrm{~b}$ and $9 \mathrm{e}, 9 \mathrm{~g}$ is a non-convex optimization problem.

\section{Proposed Solution}

First, we define the semi-definite beamforming matrix $\mathbf{V}_{l}=$ $\mathbf{v}_{l} \mathbf{v}_{l}^{H}$ and $\mathbf{W}_{l}=\mathbf{w}_{l} \mathbf{w}_{l}^{H}$. Accordingly, the rank of $\mathbf{V}_{l}$ and $\mathbf{W}_{l}$ should be one, namely $\operatorname{rank}\left(\mathbf{V}_{l}\right)=1$ and $\operatorname{rank}\left(\mathbf{W}_{l k}\right)=1$. Via 
introducing auxiliary variables $a_{l k}, b_{l k}$ and $c_{l k}$, 9) can be recast as the following SDP optimization problem

$$
\max _{\left\{\mathbf{V}_{l}, \mathbf{W}_{l, k}, \beta_{l k}, a_{l k}, b_{l k}, c_{l k}\right\}} \sum_{l=1}^{L} \sum_{K=1}^{K} B_{\mathrm{mm}} \log \left(1+a_{l k}\right)
$$

s.t. $a_{l k} \leq \gamma_{l k}^{\prime}, \forall l, k$,

$$
\begin{aligned}
& \sum_{j=1}^{L} \sum_{i=1}^{K} \operatorname{Tr}\left(\mathbf{G}_{j l k} \mathbf{W}_{j i}\right)+B_{\mathrm{mm}} \delta^{2} \geq b_{l k}, \forall l, k \\
& b_{l k}\left(1-\beta_{l k}\right) \geq E_{l k}^{\min } / \eta, \forall l, k \\
& \sum_{l=1}^{L} \operatorname{Tr}\left(\mathbf{V}_{l}\right) \leq P_{\mathrm{CP}}^{\max } \\
& \sum_{k=1}^{K} \mathbf{W}_{l k}(m, m) \leq P_{l, m}^{\max }, \forall l, m, \\
& \sum_{k=1}^{K} B_{\mathrm{mm}} \log \left(1+\gamma_{l k}^{\prime}\right) \leq c_{l}, \forall l
\end{aligned}
$$

$c_{l} \leq B_{\mathrm{mc}} \log \left(1+\frac{\operatorname{Tr}\left(\mathbf{H}_{l m} \mathbf{V}_{l}\right)}{\sum_{j \neq l}^{L} \operatorname{Tr}\left(\mathbf{H}_{l m} \mathbf{V}_{j}\right)+B_{\mathrm{mc}} \delta^{2}}\right), \forall l, m$,

$\operatorname{rank}\left(\mathbf{V}_{l}\right)=1, \operatorname{rank}\left(\mathbf{W}_{l k}\right)=1, \forall l, k$,

$\mathbf{V}_{l} \geq \mathbf{0}, \mathbf{W}_{l k} \geq \mathbf{0}, \forall l, k$,

where $\gamma_{l k}^{\prime}=\frac{\operatorname{Tr}\left(\mathbf{G}_{l k} \mathbf{W}_{l k}\right)}{\sum_{i \neq k}^{K} \operatorname{Tr}\left(\mathbf{G}_{l l k} \mathbf{W}_{l i}\right)+\sum_{j \neq 1}^{L} \sum_{i=1}^{K} \operatorname{Tr}\left(\mathbf{G}_{j k} \mathbf{W}_{j i}\right)+B_{\mathrm{mm}} \delta^{2}+\delta_{u}^{2} / \beta_{l k}}, \mathbf{G}_{j l k}=$ $\mathbf{g}_{j l k}^{H} \mathbf{g}_{j l k}, \mathbf{H}_{l m}=\sum_{l m}^{\mu} \mathbf{h}_{l m}^{H} \operatorname{Tr}\left(\mathbf{G}_{m l} \mathbf{W}_{l}\right.$

One can observe that $(10)$ is still a non-convex optimization problem due to non-convex constraints $(10 \mathrm{~b}),(10 \mathrm{~d}),(10 \mathrm{~g})$, (10h) and rank-one constraint (10i). Next, we will transform them into the convex ones by advanced approximated technologies. We first introduce auxiliary variables $\xi_{l k}$ and $\epsilon_{l k}$, and (10b) can be split into the following constraints

$$
\begin{aligned}
a_{l k} \xi_{l k} & \leq \operatorname{Tr}\left(\mathbf{G}_{l l k} \mathbf{W}_{l k}\right), \forall l, k, \\
\xi_{l k} & \geq \Gamma_{l k}+B_{\mathrm{mm}} \delta^{2}+\delta_{u}^{2} \epsilon_{l k}, \forall l, k, \\
\epsilon_{l k} & \geq 1 / \beta_{l k}, \forall l, k,
\end{aligned}
$$

where $\Gamma_{l k}=\sum_{i \neq k}^{K} \operatorname{Tr}\left(\mathbf{G}_{l l k} \mathbf{W}_{l i}\right)+\sum_{j \neq l}^{L} \sum_{i=1}^{K} \operatorname{Tr}\left(\mathbf{G}_{j l k} \mathbf{W}_{j i}\right)$. In addition, the upper bound of $a_{l k} \xi_{l k}$ can be expressed as

$$
a_{l k}^{[n]} \xi_{l k}^{2} / 2 \xi_{l k}^{[n]}+\xi_{l k}^{[n]} a_{l k}^{2} / 2 a_{l k}^{[n]} \geq a_{l k} \xi_{l k}, \forall l, k,
$$

where $a_{l k}^{[n]}$ and $\xi_{l k}^{[n]}$, respectively, denote the values of $a_{l k}^{[n]}$ and $\xi_{l k}^{[n]}$ at the $n$th iteration, and thus 11 a can be formulated into the following convex constraint

$$
a_{l k}^{[n]} \xi_{l k}^{2} / 2 \xi_{l k}^{[n]}+\xi_{l k}^{[n]} a_{l k}^{2} / 2 a_{l k}^{[n]} \leq \operatorname{Tr}\left(\mathbf{G}_{l l k} \mathbf{W}_{l k}\right), \forall l, k .
$$

Next, we can transform (10d) and (11c) into the following linear matrix inequality (LMI) constraints

$$
\left[\begin{array}{cc}
b_{l k} & \sqrt{E_{l k}^{\min } / \eta} \\
\sqrt{E_{l k}^{\min } / \eta} & \left(1-\beta_{l k}\right)
\end{array}\right] \geq \mathbf{0},\left[\begin{array}{cc}
\epsilon_{l k} & 1 \\
1 & \beta_{l k}
\end{array}\right] \geq \mathbf{0}, \forall l, k .
$$

To handle 10g), we introduce auxiliary variables $d_{l k}, v_{l k}, \tau_{l k}$, and split it into the following constraints

$$
\begin{aligned}
c_{l} & \geq \sum_{k=1}^{K} B_{\mathrm{mm}} \log \left(1+d_{l k}\right), \forall l, \\
v_{l k}^{2} & \leq d_{l k}\left(\Gamma_{l k}+B_{\mathrm{mm}} \delta^{2}+\delta_{u}^{2} \tau_{l k}\right), \forall l, k, \\
\tau_{l k} \beta_{l k} & \leq 1, \forall l, k, \\
v_{l k}^{2} & \geq \phi_{l k}, \forall l, k, \\
\phi_{l k} & \geq \operatorname{Tr}\left(\mathbf{G}_{l l k} \mathbf{W}_{l k}\right), \forall l, k .
\end{aligned}
$$

By first-order Taylor approximation technique, we have $\log (1+$ $\left.d_{l k}\right) \approx \log \left(1+d_{l k}^{[n]}\right)+\left(d_{l k}-d_{l k}^{[n]}\right) /\left(1+d_{l k}^{[n]}\right)$, where $d_{l k}^{[n]}$ denotes the value of $d_{l k}$ at the $n$th iteration. Then, (15a) can be transformed into the following convex constraint:

$$
c_{l} \geq \sum_{k=1}^{K} B_{\mathrm{mm}}\left(\log \left(1+d_{l k}^{[n]}\right)+\frac{d_{l k}-d_{l k}^{[n]}}{1+d_{l k}^{[n]}}\right), \forall l,
$$

where $d_{l k}^{[n]}$ denote the value of $d_{l k}$ at the $n$th iteration. $15 \mathrm{~b}$ can be reformulated the following LMI constraint

$$
\left[\begin{array}{cc}
d_{l k} & v_{l k} \\
v_{l k} & \Gamma_{l k}+B_{\mathrm{mm}} \delta^{2}+\delta_{u}^{2} \tau_{l k}
\end{array}\right] \geq \mathbf{0}, \forall l, k .
$$

Similar to (12) and 13), 15c can be approximated as the following convex constraint

$$
\tau_{l k}^{[n]} \beta_{l k}^{2} / 2 \beta_{l k}^{[n]}+\beta_{l k}^{[n]} \tau_{l k}^{2} / 2 \tau_{l k}^{[n]} \leq 1, \forall l, k .
$$

where $\tau_{l k}^{[n]}$ and $\beta_{l k}^{[n]}$, respectively, denote the values of $\tau_{l k}^{[n]}$ and $\beta_{l k}^{[n]}$ at the $n$th iteration. In addition, according to the first-order Taylor approximation, $v_{l k}^{2}$ can be expressed as

$$
v_{l k}^{2} \geq 2 v_{l k}^{[n]} v_{l k}-\left(v_{l k}^{[n]}\right)^{2}, \forall l, k,
$$

and we can transform (15d) as the following convex constraint

$$
2 v_{l k}^{[n]} v_{l k}-\left(v_{l k}^{[n]}\right)^{2} \geq \phi_{l k}, \forall l, k .
$$

Finally, we need to handle with 10h. By introducing auxiliary variables $\lambda_{l m}, \omega_{l m}, 10 \mathrm{~h}$ can be split into as

$$
\begin{aligned}
c_{l} & \leq B_{\mathrm{mc}} \log \left(1+\lambda_{l m}\right), \forall l, m, \\
\lambda_{l m} \omega_{l m} & \leq \operatorname{Tr}\left(\mathbf{H}_{l m} \mathbf{V}_{l}\right), \forall l, m, \\
\omega_{l m} & \geq \sum_{j \neq l}^{L} \operatorname{Tr}\left(\mathbf{H}_{l m} \mathbf{V}_{j}\right)+B_{\mathrm{mc}} \delta^{2}, \forall l, m .
\end{aligned}
$$

One can observe that only (21b) is non-convex constraint. Similar to the previous method, we directly transform (21b) into the following convex constraint

$$
\lambda_{l m}^{[n]} \omega_{l m}^{2} / 2 \omega_{l m}^{[n]}+\omega_{l m}^{[n]} \lambda_{l m}^{2} / 2 \lambda_{l m}^{[n]} \leq \operatorname{Tr}\left(\mathbf{H}_{l m} \mathbf{V}_{l}\right), \forall l, m .
$$

Now, the only obstacle is the rank-one constraint (10i). By SDP relaxation, i.e., removing (10i), we can obtain the following convex relaxed SDP optimization problem

$\max _{\left\{\mathbf{V}_{l}, \mathbf{W}_{l, k}, \beta_{l k}, a_{l k}, b_{l k}, c_{l k}, \xi_{k}, \epsilon_{l}, d_{l k}, v_{l k}, \tau_{l k}, \lambda_{l m}, \omega_{l m}\right\}} \sum_{l=1}^{L} \sum_{K=1}^{K} B_{\mathrm{mm}} \log \left(1+a_{l k}\right)$

s.t. $10 \mathrm{c},(10 \mathrm{e}),(10 \mathrm{f}), 10 \mathrm{j}),(11 \mathrm{~b}), 13), 14), 15 \mathrm{e}, 116$,

(17), (18), (20), 21a), 21c), (22).

Problem (23) can be solved by standard convex optimization technique, e.g., interior-point method [18]. On this basis, to obtain the solution of the problem [10], we need to iteratively solve (23). Specifically, starting from an initial feasible solution, we update $\left\{a_{l k}^{[n]}, \xi_{l k}^{[n]}, d_{l k}^{[n]}, \tau_{l k}^{[n]}, \beta_{l k}^{[n]}, v_{l k}^{[n]}, \lambda_{l m}^{[n]}, \omega_{l m}^{[n]}\right\}$ iteratively by solving (23) using the obtained results from the previous iteration. The above procedure is carried out until convergence. We summarize the above iterative scheme in Algorithm 1. To evaluate the characteristic of rank-one for the obtained solutions, we perform 1000 times random trials, and the number of 


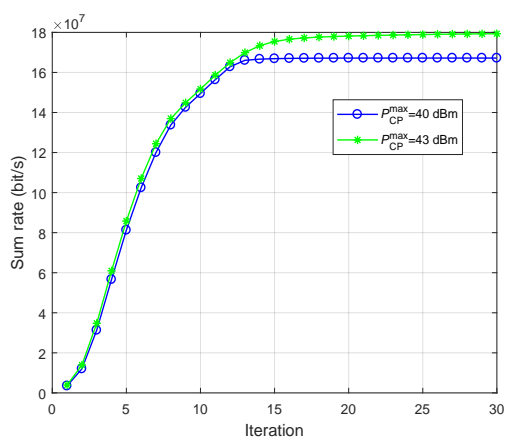

(a)

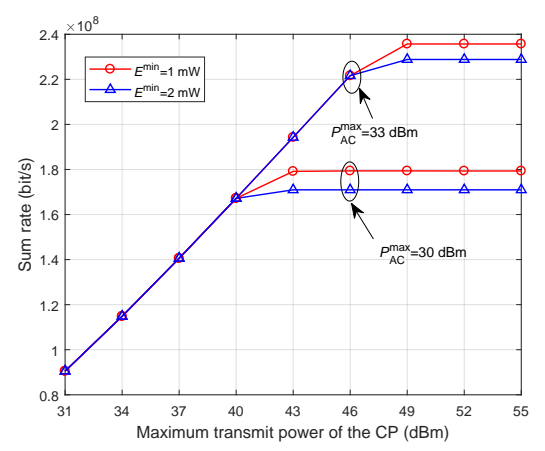

(b)

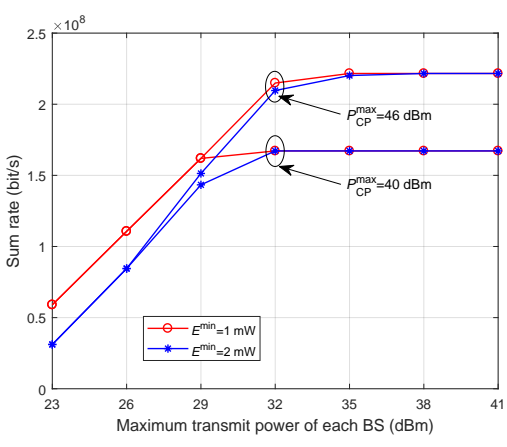

(c)

Fig. 1: Sum rate versus (a) iterations, (b) maximum transmit power of the CP, and (c) maximum transmit power of each BS.

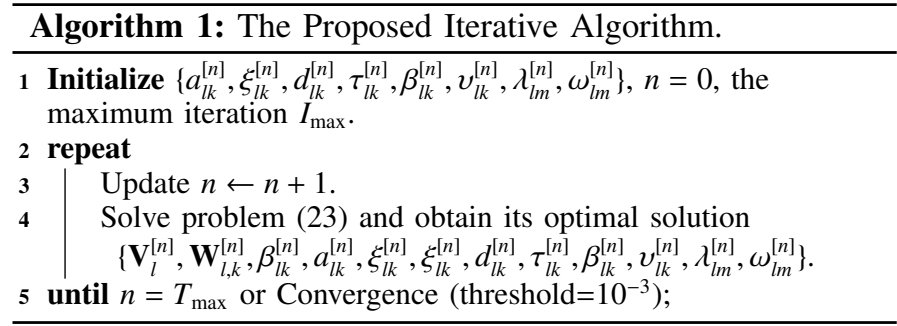

rank-one solutions is 996 (99.6\%), which shows the efficiency of the proposed Algorithm 1. Meanwhile, when the obtained solution does not satisfy the rank-one characteristic, we propose a randomization method to obtain the rank-one solution, and the detailed procedure can be found in Appendix.

Next, we discuss the effectiveness of the proposed algorithm. To obtain the solutions of the original non-convex problem (9), we need to iteratively solve the convex problem (23). The optimal solutions of (23) can be obtained at each iteration, since it is a convex optimization problem. Moreover, iteratively solving (23) will increase or at least maintain the value of the objective function in 23] [19]. Due to the limited transmit power, the objective function of (23) will be a monotonically non-decreasing sequence with an upper bound, which converges to a stationary solution that is at least locally optimal.

Now, we analyze the complexity of the proposed algorithm. Given an iterative accuracy $\varsigma$, the number of iterations is on the order $\sqrt{\Delta} \ln (1 / \varsigma)$, where $\Delta=14 K L+4 M L+M K L+N L+L+1$ denotes the barrier parameter related to the constraints [20]. In addition, (23) includes $4 K L+3 M L+L+1$ liner constraints, $3 K L$ two-dimensional LMI constraints, $L N$-dimensional LMI constraint, $K L M$-dimensional LMI constraints and $2 K L+M L$ second order cone constraints. Therefore, the total complexity of solving 23 is given by $O\left(z \sqrt{\Delta} \ln (1 / \varsigma)\left(z_{1}+z_{2} z+z_{3}+z^{2}\right)\right)$, where $z=O\left(N^{2} L+K L M^{2}\right)$ and $N^{2} L+K L M^{2}$ denotes the number of decision variables, $z_{1}=28 K L+3 M L+N^{3} L+$ $M^{3} K L+L+1, z_{2}=15 K L+3 M L+N^{2} L+M^{2} K L+L+1$, and $z_{3}=K L\left((M+2)^{2}+(N+2)^{2}\right)+4 K L$.

\section{Numerical Results}

In this section, numerical results are provided to evaluate the performance of our proposed algorithm. We assume that there are $L=2 \mathrm{BS}$ clusters, and each cluster includes $M=3 \mathrm{BSs}$ and $K=2$ users. The CP is equipped with $N=8$ antennas. We assume that all users and BSs are uniformly distributed within a circular cell with $40 \mathrm{~m}$ radius. The distance between the CP and the BS cluster center is $300 \mathrm{~m}$. The mmWave bandwidth and microwave bandwidth are assumed $B_{\mathrm{mm}}=40 \mathrm{MHz}$ and $B_{\mathrm{mc}}=20 \mathrm{MHz}$, respectively. The path loss is modeled as $69.7+$ $24 \log _{10}(d) \mathrm{dB}$ at mmWave frequency and $38+30 \log _{10}(d) \mathrm{dB}$ at microwave frequency, where $d$ in meter is the distance [21]. The noise variance is set as $-174 \mathrm{dBm} / \mathrm{Hz}$, and the noise power caused by the ID at the users is $-100 \mathrm{dBm}$. For simplicity, we set the minimum harvested energy for each user to be the same, and denote it by $E^{\mathrm{min}}$. Meanwhile, the maximum transmit power for each BS is also set the same, and denoted by $P_{\mathrm{AC}}^{\max }$. The energy conversion efficiency is set to $\eta=0.8$.

Fig. 1(a) shows the convergence performance of our proposed algorithm, where $E_{l k}^{\min }=1 \mathrm{~mW}$ and $P_{\mathrm{AC}}^{\max }=30 \mathrm{dBm}$. One can observe that the sum rate converges after 15 iterations for $P_{\mathrm{CP}}^{\max }=40 \mathrm{dBm}$, and about 20 iterations for $P_{\mathrm{CP}}^{\max }=43 \mathrm{dBm}$. Meanwhile, as expected, the sum rate is high when the CP's allowable transmit power is higher. This is because a higher CP's transmit power can provide a larger fronthaul rate.

Fig. 1(b) shows the sum rate versus maximum transmit power of the $\mathrm{CP}$ under different $E_{l k}^{\min }$ and $P_{\mathrm{AC}}^{\max }$. Under all considered conditions, the sum rate first increases with $P_{\mathrm{CP}}^{\max }$, and then saturates. In addition, although improving $P_{\mathrm{CP}}^{\max }$ can increase the fronthaul rate, this does not necessarily lead to the growth of the sum rate, since the latter is also affected by the transmit power of the BSs. For example, the sum rate has reached the maximum when $P_{\mathrm{CP}}^{\max }=43 \mathrm{dBm}$ and $P_{\mathrm{AC}}^{\max }=30 \mathrm{dBm}$, and it keeps a constant even for a higher $P_{\mathrm{CP}}^{\mathrm{max}}$. Furthermore, we can find when $P_{\mathrm{CP}}^{\max }$ is relatively low, the sum rate is the same for all considered conditions. This is because that the fronthaul rate determines the sum rate for a low $P_{\mathrm{CP}}^{\max }$, while the BS's transmit power can simultaneously satisfy the requirement of harvested energy and maximum fronthaul rate. However, as $P_{\mathrm{CP}}^{\max }$ increases, the access link rate determines the sum rate 
due to the limited transmit power of each BS. As a result, the sum rate is lower for a large $E_{l k}^{\min }$.

We plot how the sum rate varies with $P_{\mathrm{AC}}^{\max }$ in Fig. 1(c), where we set different $E_{l k}^{\min }$ and $P_{\mathrm{CP}}^{\max }$ for comparison. Similar to Fig. 1(b) the sum rate first increases and then remains stable when $P_{\mathrm{BS}}^{\max }$ increases. In fact, when $P_{\mathrm{BS}}^{\max }$ is low, the BSs first need to satisfy the requirement of each user's harvested energy and then the remaining power can be used to transform data. Nonetheless, when $P_{\mathrm{BS}}^{\max }$ is higher, the BSs have enough power to support the requirement of each user's harvested energy and maximum fronthaul rate provided by the $\mathrm{CP}$. The reasons are the same with that Fig. 1(b)

\section{Conclusion}

In this paper, we have investigated the joint beamforming and power splitting design problem in a BS cluster-based SWIPT C-RAN with multicast fronthaul. We have proposed a joint optimization algorithm of the multicast fronthaul beamforming, cooperative access beamforming and power split ratios to maximize the sum rate of the system. Meanwhile, we have analyzed the solution profile. Simulation results have verified the effectiveness of our proposed algorithm, and shown the effect of the CP's and BSs' transmit power on the sum rate.

\section{APPENDIX}

Let $\mathbf{V}_{l}^{*}$ and $\mathbf{W}_{l k}^{*}$ denote the obtained solutions of problem (9p) via our proposed algorithm. If $\operatorname{rank}\left(\mathbf{V}_{l}^{*}\right)=1 \operatorname{and} \operatorname{rank}\left(\mathbf{W}_{l k}^{*}\right)=1$, they can be respectively expressed as $\mathbf{V}_{l}^{*}=\theta_{l} \mathbf{v}_{l}^{*}\left(\mathbf{v}_{l}^{*}\right)^{H}$ and $\mathbf{W}_{l k}^{*}=\theta_{l k} \mathbf{w}_{l k}^{*}\left(\mathbf{w}_{l k}^{*}\right)^{H}$ by using eigenvalue decomposition (EVD) method, and the optimal beamforming can be directly obtained as $\mathbf{v}_{l}^{o}=\sqrt{\theta_{l}} \mathbf{v}_{l}^{*}$ and $\mathbf{w}_{l k}^{o}=\sqrt{\theta_{l k}} \mathbf{w}_{l k}^{*}$. Otherwise, we adopt the randomization technique method (see, i.e., [22]) to obtain the rank-one $\mathbf{V}_{l}^{*}$ and $\mathbf{W}_{l k}^{*}$. Specifically, applying the EVD technique, we decompose $\mathbf{V}_{l}^{*}=\mathbf{X}_{l} \mathbf{D}_{l}\left(\mathbf{X}_{l}\right)^{H}$ and $\mathbf{W}_{l k}^{*}=\mathbf{Y}_{l k} \boldsymbol{\Omega}_{l k}\left(\mathbf{Y}_{l k}\right)^{H}$. Then, by introducing the random vector $\mathbf{s}^{[n]} \sim \operatorname{CN}(\mathbf{0}, \mathbf{I})$ and $\mathbf{u}^{[n]} \sim \mathcal{C N}(\mathbf{0}, \mathbf{I})$, we form the $n$th candidate beamforming vector as $\mathbf{v}_{l}^{[n]}=\mathbf{X}_{l} \mathbf{D}_{l}^{1 / 2} \mathbf{s}^{[n]}$ and $\mathbf{w}_{l k}^{[n]}=\mathbf{Y}_{l k} \boldsymbol{\Omega}_{l k}{ }^{1 / 2} \mathbf{u}^{[n]}$, respectively. As a result, we have $\mathbb{E}\left\{\mathbf{v}_{l}^{[n]}\left(\mathbf{v}_{l}^{[n]}\right)^{H}\right\}=\mathbf{V}_{l}^{*}$ and $\mathbb{E}\left\{\mathbf{w}_{l k}^{[n]}\left(\mathbf{w}_{l k}^{[n]}\right)^{H}\right\}=\mathbf{V}_{l k}^{*}$. Finally, we substitute the $n$th candidate beamforming vectors $\mathbf{v}_{l}^{[n]}$ and $\mathbf{w}_{l}^{[n]}$ into problem $(9)$ and reformulate the following optimization problem:

$$
\begin{aligned}
\max _{\left\{c_{l}^{[n]}, t_{l k}^{[n]}, \beta_{l k}^{[n]}\right\}} & \sum_{l=1}^{L} \sum_{K=1}^{K} R_{l k}^{\mathrm{AC}[n]} \\
\text { s.t. } & E_{l k}^{[n]} \geq E_{l k}^{\min }, \forall l, k, \\
& \sum_{l=1}^{L} c_{l}^{[n]}\left\|\mathbf{v}_{l}^{[n]}\right\|^{2} \leq P_{\mathrm{CP}}^{\max }, \forall l, \\
& \sum_{k=1}^{K} t_{l k}^{[n]}\left|\mathbf{w}_{l, k}^{[n]}(m)\right|^{2} \leq P_{l, m}^{\max }, \forall l, m, \\
& \sum_{k=1}^{K} R_{l k}^{\mathrm{AC}[n]} \leq R_{l}^{\mathrm{FH}[n]}, \forall l,
\end{aligned}
$$

where $c_{l}^{[n]}$ and $t_{l k}^{[n]}$ are coefficients, $R_{l k}^{\mathrm{AC}[n]}=B_{\mathrm{mm}} \log \left(1+\gamma_{l k}^{[n]}\right)$,

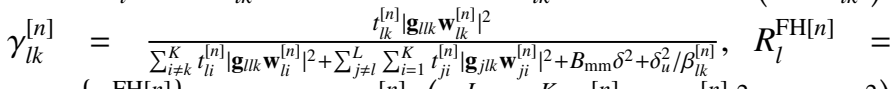
$\min _{m \in \mathcal{M}}\left\{R_{l m}^{\mathrm{FH}[n]\}}, E_{l k}=\eta\left(1-\beta_{l k}^{[n]}\right)\left(\sum_{j=1}^{L} \sum_{i=1}^{K} t_{j i}^{[n]}\left|\mathbf{g}_{j l k} \mathbf{w}_{j i}^{[n]}\right|^{2}+B_{\mathrm{mm}} \delta^{2}\right)\right.$ and $R_{l m}^{\mathrm{FH}[n]}=B_{\mathrm{mc}} \log \left(1+\frac{c_{l}^{[n]}\left|\mathbf{h}_{l m} \mathbf{v}_{l}^{[n]}\right|^{2}}{\sum_{j \neq l}^{L}\left[c_{j}^{(n)}\left|\mathbf{h}_{l m} \mathbf{v}_{j}^{n n}\right|^{2}+B_{\mathrm{m}} \delta^{2}\right.}\right)$. It can be observed that problem (24) can be solved using the iterative method proposed in Section III. Finally, we execute problem (24) repetitively for multiple different candidate vectors and select the optimal $c_{l}^{[n] *}$ and $t_{l k}^{[n] *}$ that owns the maximum sum rate, i.e., $\mathbf{v}_{l}^{\mathrm{o}}=\sqrt{c_{l}^{[n] *}} \mathbf{v}_{k}^{[n]}$ and $\mathbf{w}_{l k}^{\mathrm{o}}=\sqrt{t_{l k}^{[n] *}} \mathbf{w}_{l k}^{[n]}$.

\section{REFERENCES}

[1] C.-L. I, J. Huang, et al., "Recent progress on C-RAN centralization and cloudification," IEEE Access, vol. 2, pp. 1030-1039, 2014.

[2] J. Li, et al., "Energy-efficient joint congestion control and resource optimization in heterogeneous cloud radio access networks," IEEE Trans. Veh. Technol., vol. 65, no. 12, pp. 9873-9887, Dec. 2016.

[3] S.-H. Park, et al., "Joint decompression and decoding for cloud radio access networks," IEEE Signal Process. Lett., vol. 20, no. 5, pp. 503506, May 2013

[4] B. Dai, et al., "Optimized base-station cache allocation for cloud radio access network with multicast backhaul," IEEE J. Sel. Areas Commun., vol. 36, no. 8, pp. 1737-1750, Aug. 2018.

[5] W. Hao, et al., "Price-based resource allocation in massive MIMO HCRANs with limited fronthaul capacity," IEEE Trans. Wireless Commun., vol. 17, no. 11, pp. 7691-7703, Nov. 2018.

[6] B. Hu, et al., "Joint fronthaul multicast beamforming and user-centric clustering in downlink C-RANs," IEEE Trans. Wireless Commun., vol. 16, no. 8, pp. 5395-5409, Aug. 2017.

[7] S. Bi, et al., "Wireless powered communication: opportunities and challenges," IEEE Commun. Magazine, vol. 53, no. 4, pp. 117-125, Apr. 2015.

[8] Q. Shi, et al., "Energy efficiency optimization for MISO SWIPT systems with zero-forcing beamforming," IEEE Trans. Signal Processing, vol. 64, no. 4, pp. 842-854, Feb. 2016.

[9] S. Lee, et al., "Collaborative wireless energy and information transfer in interference channel," IEEE Trans. Wireless Commun., vol. 14, no. 1, pp. 545-557, Sep. 2015.

[10] W. Hao, et al., "Energy-efficient hybrid precoding design for integrated multicast-unicast millimeter wave communications with SWIPT," IEEE Trans. Veh. Technol., vol. 68, no. 11, pp. 10956-10968, Nov. 2019.

[11] C. Qin, et al., "Joint rate maximization of downlink and uplink in multiuser MIMO SWIPT systems," IEEE Access, vol. 5, pp. 3750-3762, 2017.

[12] M. Zhao, et al., "Joint transceiver design for full-duplex cloud radio access networks with SWIPT," IEEE Trans. Wireless Commun., vol. 16, no. 9, pp. 5644-5658, Sep. 2017.

[13] W. Hao et al., "Beamforming design in SWIPT-based joint multicastunicast mmWave massive MIMO with lens-antenna array," IEEE Wireless Commun. Lett., vol. 8, no. 4, pp. 1124-1128, Aug. 2019.

[14] Y. Ma, et al., "Optimization of simultaneous wireless information and power transfer in cloud radio access networks," in Proc. IEEE 83rd VTC, Nanjing, 2016, pp. 1-5.

[15] Y. Zhao, et al., "Energy-efficient sub-carrier and power allocation in cloud-based cellular network with ambient RF energy harvesting," IEEE Access, vol. 5, pp. 1340-1352, 2017.

[16] Z. Chen, et al., "Beamforming design for max-min fair SWIPT in green cloud-RAN with wireless fronthaul," in Proc. IEEE GLOBECOM, Abu Dhabi, 2018, pp. 1-6.

[17] R. Horst and N. V. Thoai, "DC programming: overview," J. of Optimization Theory and Application, vol. 103, no. 1, pp. 1-43, 1999.

[18] S. Boyd and L. Vandenberghe, Convex Optimization. Cambridge University Press, 2009.

[19] Q. Zhang, et al., "Robust beamforming for nonorthogonal multiple-access systems in MISO channels," IEEE Trans. Veh. Technol., vol. 65, no. 12, pp. 10231-10236, Dec. 2016.

[20] K.-Y. Wang, et al., "Outage constrained robust transmit optimization for multiuser MISO downlinks: Tractable approximations by conic optimization,' IEEE Trans. Signal Process., vol. 62, no. 21, pp. 5690-5705, Nov. 2014.

[21] R. G. Stephen and R. Zhang, "Joint millimeter-wave fronthaul and OFDMA resource allocation in ultra-dense CRAN," IEEE Trans. Wireless Commun., vol. 65, no. 3, pp. 1411-1423, Mar. 2017.

[22] N. D. Sidiropoulos, et al., "Transmit beamforming for physical-layer multicasting," IEEE Trans. Signal Process., vol. 54, no. 6, pp. 2239-2251, Jun. 2006. 